\title{
Objective gustatory and olfactory dysfunction in COVID-19 patients: a prospective cross-sectional study
}

\author{
Thirza Singer-Cornelius $^{1} \mathbb{D} \cdot$ Julian Cornelius ${ }^{2} \cdot$ Michael Oberle $^{3} \cdot$ Frank U. Metternich $^{1} \cdot$ Steffi Johanna Brockmeier ${ }^{1}$
}

Received: 20 October 2020 / Accepted: 24 December 2020 / Published online: 20 January 2021

(c) The Author(s), under exclusive licence to Springer-Verlag GmbH, DE part of Springer Nature 2021

\begin{abstract}
Purpose To determine the prevalence of objective gustatory (GD) and olfactory (OD) dysfunction in COVID-19 patients. Methods This is a prospective, cross-sectional study of 51 COVID-19 positive patients diagnosed using RT-PCR-based testing. Of these study participants, 41 reported having present GD and OD at the time of enrollment and ten patients were without symptomatic OD and GD. All participants were objectively tested for OD by Brief Smell Identification Test (BSIT) and for GD by Burghart taste strip test, which were mailed to the participants. The subjective presence and severity of COVID-19 symptoms of smell loss, loss of taste, nasal obstruction, rhinorrhea/mucus production, fever, cough and shortness of breath were also assessed.

Results Of the 41 patients with GD and OD, only 25.6\% (10/39; $p \leq 0.0001)$ objectively presented GD and 39.1\% (16/41; $p \leq 0.0001)$ OD at the time of their subjective dysfunction. Regarding GD, 23.1\% (9/39) suffered from total hypogeusia, $2.6 \%(1 / 39)$ from ageusia. A significant loss of sour (33.3\% (13/39)) and salty taste (17.9\% (7/39)) could be recognized. Only $10.3 \%$ (4/39) showed a reduction in sweet and bitter taste. Concerning OD, 9.8\% (4/41) showed a deficit relative to younger age in the BSIT and $29.3 \%$ (12/41) results abnormal relative to age.

Conclusion Subjective and objective findings in GD and OD differ significantly. Most patients suffering from objective dysgeusia present a deficit in sour and salty taste.
\end{abstract}

Trial registration number DRKS00021516; 22/04/2020.

Keywords Gustatory dysfunction · Olfactory dysfunction · COVID-19 · Taste loss · Smell loss

\section{Introduction}

SARS-CoV-2 is a novel coronavirus causing the coronavirus disease, firstly detected in 2019 (COVID-19), which is currently causing a pandemic affecting over 200 countries. Besides unspecific symptoms, olfactory dysfunction (OD) and gustatory dysfunction (GD) have been found to be surprisingly prevalent in the COVID-19 disease course $[1,2]$. GD in COVID-19 has largely been attributed to OD, although it is possible that COVID-19 may be directly

Thirza Singer-Cornelius

thirza.singer@ksa.ch

1 Klinik für Hals-Nasen-Ohrenheilkunde, Hals- und Gesichtschirurgie, Kantonsspital Aarau, Aarau, Switzerland

2 Departement Chirurgie, Luzerner Kantonsspital, Lucerne, Switzerland

3 Institut für Labormedizin, Kantonsspital Aarau, Aarau, Switzerland associated with dysfunction of taste receptor cells in the oral cavity. It has been shown that angiotensin-converting enzyme 2 (ACE2), the required entry receptor for SARS$\mathrm{CoV}-2$, is expressed on oral epithelium cells and in salivary glands. High expression of ACE2 in the dorsum linguae represents a potentially causal mechanism for GD by SARSCoV-2 through direct infection of taste receptor cells and thus influence of stimulus transmission [3]. Furthermore, a large quantity of ACE2 was found in nasal epithelial cells, especially in goblet/secretory cells and ciliated cells [4]. Earlier detected coronavirus strains, such as SARS-CoV and human coronavirus OC43, are shown to invade the central nerve system via the olfactory neuroepithelium to the olfactory bulb to the piriform cortex and brain stem, which could indicate an affection of the central olfactory pathway, causing a smell deficit $[5,6]$.

Several studies have investigated the prevalence of subjectively reported GD and OD in COVID-19 patients. The rates for GD and OD widely range from 33.9 to $85.6 \%[1,2$, 
7]. Previously, data from our study group indicate a prevalence of patient-reported OD of $61.2 \%$ and GD of $65 \%$ [2, $8,9]$. Both sense disorders were highly correlated with each other [1,2]. Studies of objective OD determined through testing are contradictory. Moein et al. [10] reported the prevalence of OD to be even 83-98\% [10]. On the other hand, Lechien et al. [1] described a discrepancy between subjective and objective OD, leading to the assumption that subjective OD is more often reported than actually existing [11]. Compared to OD, objective testing of taste function in COVID-19 has not yet been performed.

The prevailing theory for the mechanism of GD during COVID-19 has been as a secondary consequence of OD, although the possibility of direct impact of SARS-CoV-2 on taste pathways has been proposed. Therefore, objective testing of the sense of taste would be able to distinguish GD as a secondary consequence of OD from primary GD due to dysfunction of taste sensation pathways. In this study, our objective was to assess objective GD in COVID-19 positive patients, in the context of objective OD as well as subjectively reported symptoms by patients.

\section{Materials and methods}

\section{Study population}

Ethics committee approval was obtained from both Ethikkommission Nordwest und Zentralschweiz (2020-00666) and the institutional review board of the Kantonsspital Aarau (1410.000.128). Patients positively tested for SARS-CoV-2 between March 3, 2020, and May 12, 2020, at the Kantonsspital Aarau were invited to participate in the study, and their informed consent was obtained. Inclusion criteria were positive SARS-CoV-2 RT-PCR test, age over 18 years and sufficient understanding of German language for providing informed consent. Exclusion criteria were: preexisting OD and/or GD, history of sinus operation and radio- or chemotherapy in the head and neck region as well as current stay in the intensive care unit. Patients who could not be reached within three telephone calls were not screened for inclusion.

\section{Study design}

This was a prospective, cross-sectional study. Out of 182 patients positively tested by SARS-CoV-2 RT-PCR, 139 patients provided informed consent, met the inclusion criteria mentioned above and were interviewed by questionnaire via telephone (screening phone call), as previously described $[2,8,9]$. The interview revealed a total of 85 patients, who described GD and/or OD during their course of COVID19, 49 of them with present GD and OD. Among them, 8 patients were excluded due to not fulfilling the inclusion criteria.

These 41 patients were invited to do objective olfactory and taste testing. The Brief Smell Identification Test (BSIT) and the Burghart taste test were sent to the patient by mail [12-14]. Participants were guided how to perform the tests during another phone or video-call. Only patients, who reported a persistent OD and GD when performing the test, as well as symptom onset of their OD and GD between 1 and 21 days before the screening phone call, were considered for the testing.

Ten patients out of 54, who were tested positively for COVID-19 but did not report subjective GD and OD in the whole period of their COVID-19 course of disease were recruited as a control group. Patients within the control group and 10 participants of the symptomatic group were matched for age, gender and time from the onset of GD and OD and absolving the objective testing ( \pm 1 day). Matching was performed according to age with groups consisting of a decade (20-29, 30-39, 40-49, 50-59 and $\geq 60)$. At least one match was found for each age subgroup. If possible, the exact same birth year and gender was matched.

\section{Questionnaire and objective taste and smell test}

For the screening phone call, a standardized questionnaire was used [2, 8, 9]. Demographic features such as age, gender, smoking history, history of allergic rhinitis/hay fever, chronic rhinosinusitis and asthma were raised. A qualitative classification concerning severity of the symptoms of decreased sense of smell, decreased sense of taste, nasal obstruction, rhinorrhea/nasal mucus production, fever, cough and shortness of breath of each was performed using a scale, modeled to the validated nasal symptom score (0 (none), 1 (mild), 2 (moderate), 3 (severe)) [15]. The severity of GD and OD was queried on a scale of 0 (none), 1 (mild), 2 (moderate), 3 (severe) during the test performing phone call.

For objectification of GD, taste strips by Burghart Messtechnik GmbH, Wedel, Germany, were used. This validated test contains 18 spoon-shaped strips per participant impregnated with four different concentrations of sweet, sour, salty and bitter as well as two blanks [13]. The participant was given a mixed order of the different tastes and concentrations suggested by the producer. Each strip had to be placed in the middle of the tongue. Subjects had to decide between the answer possibilities of "no taste," "sweet," "sour," "salty" and "bitter." After each taste strip, the mouth was thoroughly rinsed with water. A maximum of 16 points could be achieved. A score below the threshold value of nine points is called hypogeusia, no sensation in each of the taste qualities ageusia. Additionally, a hypogeusia (1 or false identification) or ageusia (no sensation) only for certain taste qualities can be measured. 
The Brief Smell Identification Test (BSIT) version A in German by the University of Pennsylvania (Sensonics International, Haddon Hts., NJ, USA) was used. The BSIT is a validated and reliable smell test containing a microcapsule that has to be scratched and sniffed $[12,16]$. Out of four possible answers, one answer has to be chosen by the patient. The test includes 12 different odors ( 12 points $=$ maximum $)$ and is a single-use test. The results can be labeled as "normal," "deficit relative to younger age" and "abnormal to age" according to the age-related normative tables of the BSIT manual $[12,16]$.

\section{Statistical analysis}

Statistical analysis was carried out using the software PRISM GraphPad Version 7.0 for Mac, GraphPad Software, La Jolla, California, USA, www.graphpad.com. Descriptive statistical data are expressed as mean \pm standard deviation (SD). Mann-Whitney and ANOVA tests with significance defined as $p \leq 0.05$ were used. For normality testing, Shapiro-Wilk test was applied. In order to evaluate possible correlating factors, Pearson`s correlation coefficient was calculated.

\section{Results}

\section{Patient characteristics and clinical outcome}

A total of 41 participants with GD and OD during COVID19 infection were recruited (in the following called "symptomatic patients") along with ten control participants with COVID-19 who reported no GD or OD but presented other COVID-related symptoms (in the following called "asymptomatic patients"). Patient characteristics are described in Tables 1, 2. All patients absolved the BSIT, while two of symptomatic patients did not perform the taste strips test, one due to pregnancy (contraindication of the test), one refused. The median age was $35.22 \pm 13.53$ years, 31 patients were female $(75.6 \%)$ and ten $(24.4 \%)$ male. Comorbidities included allergic rhinitis and/or hay fever $(n=14$; $34.1 \%)$, chronic rhinosinusitis $(n=1,2.4 \%)$ and bronchial asthma $(n=6 ; 14.6 \%)$. Most of the patients were nonsmokers $(n=27 ; 65.9 \%)$. In the control group, median age was $36.2 \pm 15.5$ years. Nine patients were females $(90 \%)$, one male $(10 \%)$.

Severities of symptomatic and asymptomatic patients' COVID-19 symptoms are described in Table 3. In the symptomatic cohort, $53.7 \%(22 / 41)$ reported a mild, $14.6 \%$ (6/41) a moderate and $31.7 \%$ (13/41) a severe GD. Regarding OD, $31.7 \%(13 / 41)$ claimed it as mild, $19.5 \%$ (8/41) as moderate and $48.8 \%(20 / 41)$ as severe. The majority in this group did
Table 1 Patient characteristics in the overall symptomatic cohort

\begin{tabular}{ll}
\hline & $\begin{array}{l}\text { Overall } \\
\text { symptomatic } \\
\text { cohort } \\
N=41\end{array}$ \\
& \\
\hline Age in years & $35.22( \pm 13.5)$ \\
Median $( \pm$ SD) & \\
Gender & $31(75.6 \%)$ \\
Female & $10(24.4 \%)$ \\
Male & \\
Allergic rhinitis or hay fever & $14(34.1 \%)$ \\
Yes & $27(65.9 \%)$ \\
No & \\
Chronic rhinosinusitis & $1(2.4 \%)$ \\
Yes & $40(97.6 \%)$ \\
No & \\
Any medication & $13(31.7 \%)$ \\
Yes & $28(68.3 \%)$ \\
No & \\
Bronchial asthma & $6(14.6 \%)$ \\
Yes & $35(85.4 \%)$ \\
No & \\
Smoker & $33(80.5 \%)$ \\
Active smoker & $8(19.5 \%)$ \\
\hline
\end{tabular}

not suffer from nasal obstruction (58.5\%) or nasal secretion (73.2\%).

The mean time between the beginning of COVID-19 symptoms and when the participants completed the objective olfactory and taste testing was $12.61( \pm 6.58)$ days.

\section{Subjective and objective olfactory and gustatory dysfunction}

All of the 41 patients suffered from subjective smell and taste dysfunction at the timepoint of BSIT, and taste strips test was performed. Of the patients, $34.1 \%(14 / 41)$ could not assign the GD to a certain taste quality. In the remaining cohort of 27 patients, the following taste deficits were subjectively reported: $74.1 \%$ (20/27) sweet, $77.8 \%$ (21/27) sour, 70.4\% (19/27) salty, 63\%, (17/27) bitter. Patients showed no significant difference in their subjectively reported dysfunction of the different tastes. In contrast, objective results of the Burghart taste strips test revealed that $74.4 \%(29 / 39)$ had a normal test result $(p \leq 0.0001)$, while $23.1 \%(9 / 39)$ had hypogeusia and $2.6 \%(1 / 39)$ ageusia. In further detail, the Burghart test revealed that $10.3 \%(4 / 39)$ objectively had deficits in the ability to sense "sweet," $33.3 \%$ (13/39) in the ability to sense "sour," $17.9 \%(7 / 39)$ in the ability to sense "salty" and $10.3 \%$ (4/39) in the ability to sense "bitter." 
Table 2 Patient characteristics of matched pairs

\begin{tabular}{|c|c|c|c|}
\hline & $\begin{array}{l}\text { Symptomatic cohort } \\
N=10\end{array}$ & $\begin{array}{l}\text { Asymptomatic cohort } \\
N=10\end{array}$ & $p$ values \\
\hline Age in years & & & 0.93 \\
\hline Median $( \pm$ SD) & $35.40( \pm 15.5)$ & $36.20( \pm 15.5)$ & \\
\hline Gender & & & $>0.9999$ \\
\hline Male & $1(10 \%)$ & $1(10 \%)$ & \\
\hline Female & $9(90 \%)$ & $9(90 \%)$ & \\
\hline Allergic rhinitis or hay fever & & & $>0.9999$ \\
\hline Yes & $5(50 \%)$ & $5(50 \%)$ & \\
\hline No & $5(50 \%)$ & $5(50 \%)$ & \\
\hline Chronic rhinosinusitis & & & $>0.9999$ \\
\hline Yes & $0(0 \%)$ & $0(0 \%)$ & \\
\hline No & $10(100 \%)$ & $10(100 \%)$ & \\
\hline Bronchial asthma & & & 0.30 \\
\hline Yes & $4(40 \%)$ & $0(0 \%)$ & \\
\hline No & $6(60 \%)$ & $10(100 \%)$ & \\
\hline Smoker & & & 0.65 \\
\hline Nonsmoker & $3(30 \%)$ & $8(80 \%)$ & \\
\hline Active smoker & $7(70 \%)$ & $2(20 \%)$ & \\
\hline
\end{tabular}

The difference between subjective and objective taste loss was significant for each sense of taste (sweet: $p \leq 0.0001$, sour: $p=0.001$, salty: $p \leq 0.0001$, bitter: $p \leq 0.0001)$. Results are shown in Fig. 1.

Concerning OD, only $39.1 \%$ (16/41) objectively presented an OD, thus showing a significant difference in subjective and objective findings $(p \leq 0.0001)$. A "deficit relative to younger patients" could be seen in $9.8 \%$ (4/41), a "result abnormal to age" in $29.3 \%$ (12/41).

The time from onset of the subjective OD and GD until objective testing did not differ significantly between patients with normal or pathologic results of taste test and/ or BSIT. The mean time from onset to objective testing was $12.8 \pm 6.9$ days in patients with normal taste test results and $14.1 \pm 7.8$ days in patients with pathologic test results. Concerning BSIT, the mean time in patients with normal test results was $12.4 \pm 7.3$ days and $14.3 \pm 6.4$ in patients with pathologic test results.

\section{Correlation between BSIT and taste test results}

The Pearson's correlation coefficient was calculated in order to evaluate the correlation between the taste test and BSIT. There was no significant correlation between objective GD and OD (taste strips and BSIT) $(r=0.12$; CI $95 \%$ ( -0.20 to 0.42$) ; p=0.46$ ). Furthermore, there was no significant correlation between patient characteristics shown in Tables 1, 2 and objective GD and OD (data not shown).

\section{Matched pairs analysis}

Objectively, none of the asymptomatic patients was found to have decreased sense of taste (hypogeusia or ageusia) on the Burghart test concerning total points achieved. Regarding the different taste qualities, none presented a GD in sweet and salty, $30 \%$ in sour and $20 \%$ in bitter taste quality. Overall, no significant difference could be seen between the symptomatic and asymptomatic patients for GD in all four taste qualities (Table 4).

Regarding OD, $50 \%$ of symptomatic patients had either a "deficit compared to younger age" or" abnormal test results related to age." In contrast, only $10 \%$ of patients in the control group showed a "deficit compared to younger age" $(p=0.10)$. Patients reporting OD had significantly lower points in the total BSIT score $(7.7 \pm 3.7)$ when compared to the control group $(10.6 \pm 1.35)(p=0.04)$ (Table 4, Fig. 2).

\section{Discussion}

Until now, SARS-CoV-2 is widely spreading in the whole world. Unfortunately, no vaccination or targeted therapy is still available, underlining the importance of detailed knowledge in symptoms and pathomechanisms. The prevalence of dysgeusia and dysosmia in COVID-19 patients has been discussed frequently during the past months. However, the objective extent of OD and GD and its pathophysiological mechanisms have not yet been fully revealed. 
Table 3 Clinical outcome in the survey of the total cohort

\begin{tabular}{|c|c|c|}
\hline & $\begin{array}{l}\text { Symptomatic cohort with pre- } \\
\text { sent OD and GD } \\
N=41\end{array}$ & $\begin{array}{l}\text { Asymptomatic } \\
\text { cohort for OD and } \\
\text { GD } \\
N=10\end{array}$ \\
\hline \multicolumn{3}{|c|}{ Subjective OD when performing the test } \\
\hline No dysfunction & 0 & $10(100 \%)$ \\
\hline Mild & $13(31.7 \%)$ & $0(0 \%)$ \\
\hline Moderate & $8(19.5 \%)$ & $0(0 \%)$ \\
\hline Severe & $20(48.8 \%)$ & $0(0 \%)$ \\
\hline \multicolumn{3}{|c|}{ Subjective GD when performing the test } \\
\hline No dysfunction & $0(0 \%)$ & $10(100 \%)$ \\
\hline Mild & $22(53.7 \%)$ & $0(0 \%)$ \\
\hline Moderate & $6(14.6 \%)$ & $0(0 \%)$ \\
\hline Severe & $13(31.7 \%)$ & $0(0 \%)$ \\
\hline \multicolumn{3}{|l|}{ Nasal obstruction } \\
\hline No obstruction & $24(58.5 \%)$ & $10(100 \%)$ \\
\hline Mild & $13(31.7 \%)$ & $0(0 \%)$ \\
\hline Moderate & $4(9.8 \%)$ & $0(0 \%)$ \\
\hline Severe & $0(0 \%)$ & $0(0 \%)$ \\
\hline \multicolumn{3}{|l|}{ Nasal secretion } \\
\hline No secretion & $30(73.2 \%)$ & $8(80 \%)$ \\
\hline Mild & $10(24.4 \%)$ & $0(0 \%)$ \\
\hline Moderate & $1(2.4 \%)$ & $2(20 \%)$ \\
\hline Severe & $0(0 \%)$ & $0(0 \%)$ \\
\hline \multicolumn{3}{|l|}{ Fever } \\
\hline No fever & $30(73.2 \%)$ & $10(100 \%)$ \\
\hline Mild & $7(17.1 \%)$ & $0(0 \%)$ \\
\hline Moderate & $3(7.3 \%)$ & $0(0 \%)$ \\
\hline Severe & $1(2.4 \%)$ & $0(0 \%)$ \\
\hline \multicolumn{3}{|l|}{ Coughing } \\
\hline No coughing & $21(51.2 \%)$ & $9(90 \%)$ \\
\hline Mild & $13(31.7 \%)$ & $0(0 \%)$ \\
\hline Moderate & $5(12.2 \%)$ & $1(10 \%)$ \\
\hline Severe & $2(4.9 \%)$ & $0(0 \%)$ \\
\hline \multicolumn{3}{|l|}{ Shortness of breath } \\
\hline No shortness of breath & $30(73.3 \%)$ & $8(80 \%)$ \\
\hline Mild & $8(19.5 \%)$ & $1(10 \%)$ \\
\hline Moderate & $3(7.3 \%)$ & $1(10 \%)$ \\
\hline Severe & $0(0 \%)$ & $0(0 \%)$ \\
\hline
\end{tabular}

Data concerning the prevalence of subjective smell and taste dysfunction vary between $33.9 \%$ and $88.8 \%$. Both symptoms seem to be significantly associated with each other $[1,2,7]$. Contrary to these subjective findings, only $25.6 \%(10 / 39)$ of all patients who reported a persistent taste dysfunction showed a measurable taste deficit in any kind of taste quality in our study. When considering the taste qualities in detail, the significant difference between subjective and objective taste dysfunction is even clearer. Of patients, $74.1 \%$ reported a subjective GD in sweet taste, $77.8 \%$ in sour, $70.4 \%$ in salty and $63 \%$ in bitter. In contrast, only $10.3 \%$ presented objective GD in sweet, $33.3 \%$ in sour,
$17.9 \%$ in salty and $10.3 \%$ in bitter. These results suggest that although GD and OD are often reported symptoms in a COVID-19 course of disease, the subjective perception of smell and taste dysfunction might be often overestimated. A possible reason could be that at the timepoint our study was conducted, all of the patients were already informed about their positive SARS-CoV-2 test and most knew about the possible occurrence of smell and taste dysfunction in COVID-19. Furthermore, patients in this study frequently reported having difficulties in distinguishing between the senses of smell and taste. Another difficulty specifically in our cohort might be that smell and taste are the same word 
(a)

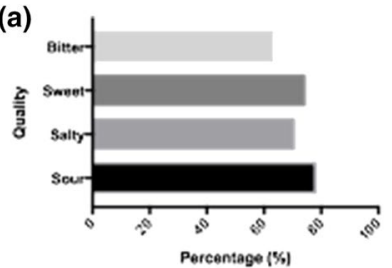

(b)

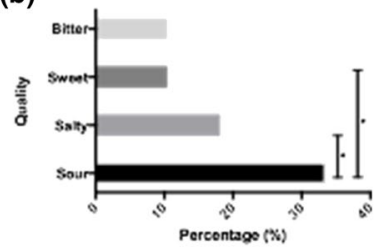

(c)

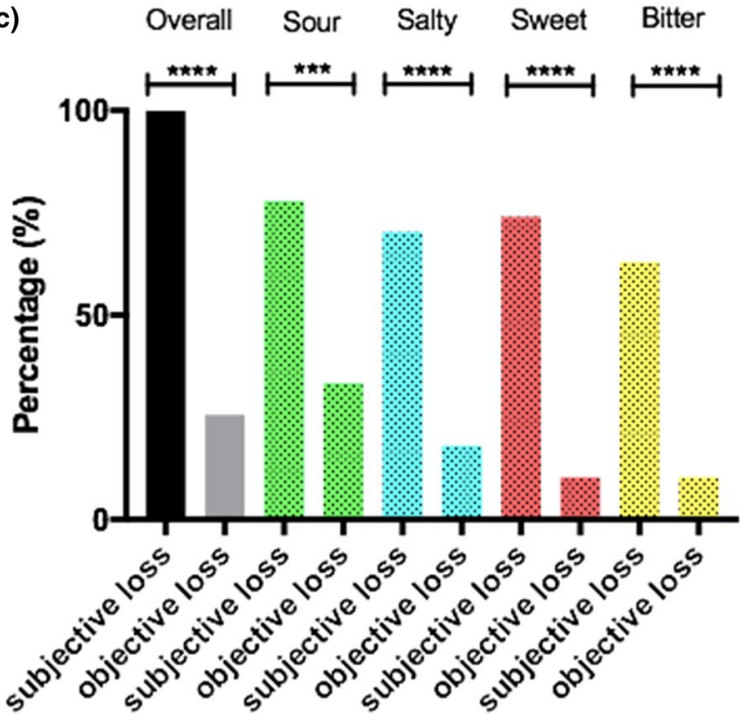

Fig. 1 Subjective (a) and objective (b) loss of each taste quality and their direct comparison (c)

in Swiss German dialect. Regarding the Burghart taste strips test, hypogeusia is assigned if only one out of four, an ageusia if none out of four filter papers of a certain taste quality in different concentrations is assorted correctly. The possible answers include sweet, sour, salty, bitter or no taste. Sometimes, patients were sure that they tasted something but could not easily assort it to the specific taste quality, thus choosing the wrong or accidentally the right answer. Therefore, to fulfill the criteria of hypogeusia in this test is difficult. The Burghart taste strips test offers a good tool for a first clinical assessment of taste loss, especially concerning ageusia; however, fine nuances of hypogeusia cannot be detected easily and could thus be also part of the reason why subjective and objective data differ in our cohort. This is also supported by the study of Wolf et al. who describe a higher accuracy of the results in the extended new version of taste strips containing six different concentrations for each taste quality [17].

In several studies, a correlation between subjective GD and OD could be recognized [1, 2]. However, our objective tests did not show a significant correlation. This supports the thesis that GD and OD in COVID-19 present two independent symptoms caused by SARS-CoV-2 and that GD is not a secondary consequence of OD.

We are the first who investigated the different taste qualities in COVID-19 patients in detail. Interestingly, our data revealed that the taste qualities "sour" (33.3\%) and "salty" (17.9\%) were significantly reduced in our patient cohort. Both taste qualities are known to be transmitted by ion channels, whereas bitter (TR2 receptor) and sweet and umami (TR1 receptor) are taste qualities transduced by a G-protein-coupled receptor [18]. In a study by Shigemura et al. 2013, a suppressive function of angiotensin II in amiloridesensitive salt taste response and enhancement of sweet taste response was seen through behavioral analysis. This effect was assumed to be mediated by AT1 receptors, which were found to be co-expressed with $\alpha \mathrm{ENaC}$ (a sodium channel, proposed to be a component of the salt taste system) and T1r3 (mediating sweet taste) [19]. Kai et al. 2020 presented a possible scheme in which the interactions of SARS-CoV-2 with ACE2, angiotensin II and RAS inhibitors are presented [24]. It was hypothesized that SARS-CoV-2 enters cells via ACE2, replicates in the host cells and degrades ACE2. This might lead to a lower degradation of angiotensin II and thus higher concentration of angiotensin II [20]. Therefore, it was assumed that the higher concentration of angiotensin II, caused by lower ACE2-levels by SARS-CoV-2 infiltration, could be the reason for the taste dysfunction of the quality salty and the comparatively good results in sweet taste.

Until now, the sour taste transduction is not yet fully revealed. A current hypothesis is that protons are shuttled into the cell via proton channels, where a cytosolic depolarization leads to a blockade of the potassium channel KIR2.1 and thus firing of APs (action potentials) [21]. In cardiologic studies, an overexpression of angiotensin II leads to an increased AT1 receptor expression and thus suppression of KIR2.1 expression in the heart [22]. This might explain the alteration of sour taste through SARSCoV-2. Of course, these explanations for the different results of each taste quality are hypothetical. Our data

Table 4 Matched pairs analysis: dysgeusia and dysosmia in symptomatic and asymptomatic cohort

\begin{tabular}{lllllll}
\hline & Sweet taste & Sour taste & Salty taste & Bitter taste & BSIT & BSIT total points \\
\hline Objective symptomatic cohort & $2 / 10(20 \%)$ & $5 / 10(50 \%)$ & $3 / 10(30 \%)$ & $0 / 10(0 \%)$ & $5 / 10(50 \%)$ & $7.7 \pm 3.7$ \\
Objective asymptomatic cohort & $0 / 10(0 \%)$ & $3 / 10(30 \%)$ & $3 / 10(0 \%)$ & $2 / 10(20 \%)$ & $1 / 10(10 \%)$ & $10.6 \pm 1.35$ \\
$p$ value & 0.47 & 0.65 & 0.21 & 0.47 & 0.10 & $\mathbf{0 . 0 4}$ \\
\hline
\end{tabular}

Significant $p$ values are printed in bold 


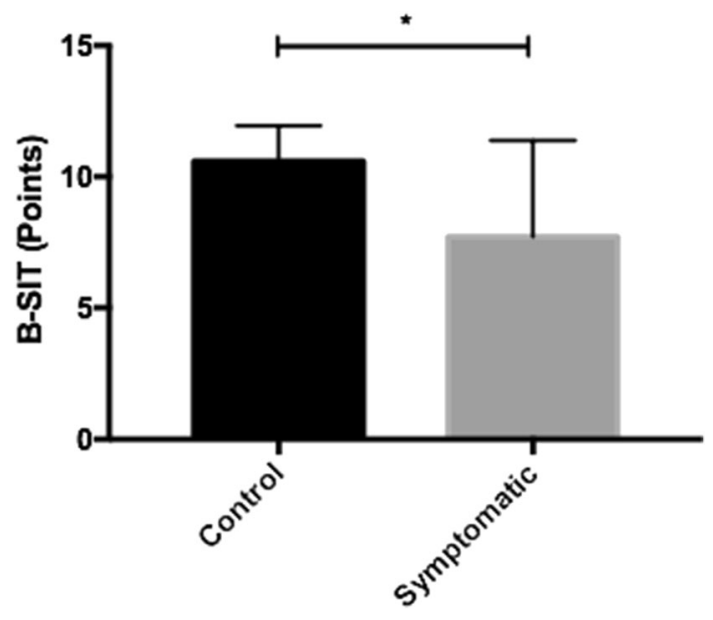

Fig. 2 Range of points achieved in the BSIT in the control group and group symptomatic for OD

suggest that the qualities sour and salty are more affected during the COVID-19 course of disease. Although the difference to sweet and bitter is significant, the results we achieved can only be seen as a trend mark due to a small cohort and limited possibilities of differentiated testing with taste strips as mentioned above. Therefore, future experimental studies are needed including bigger patient cohorts and more detailed testing methods in order to prove these hypotheses and to further investigate how SARS-CoV-2 affects the taste qualities on a molecular basis.

Concerning OD, Lechien et al. [1] reported a discrepancy between subjective and objective OD. They found that only $61.7 \%$ of all patients reporting a subjective OD actually presented a hyposmia or anosmia in their testing [11]. In accordance, our results indicate even a higher discrepancy between subjective and objective GD and OD. Only $39.1 \%$ (16/41) of patients reporting smell loss subjectively were found to have a proven smell deficit in objective testing. Yet, comparing symptomatic and asymptomatic COVID-19 positive patients for OD, the symptomatic group achieved a significantly lower total score in BSIT. A simple reason for this disparity between the study by Lechien et al. [1] and our data could be the different smell test used.

For the development of OD in the COVID-19 disease course, high expression of ACE2, which is claimed as SARSCoV-2 entry receptor, in the nasal mucosa, especially in goblet and ciliated cells could play a crucial role [4, 23]. Interestingly, also the highest viral titers of SARS-CoV-2 are detected in the nose [24]. In a study with SARS-CoV, it was assumed that SARS-CoV-2 also invades the central nervous system through the olfactory bulb, causing transneuronal spreading and consequently leading to respiratory failure $[5,25]$. Therefore, a neuronal component could be the reason for the development of OD in the course of COVID-19 infection.

The results of our study are subject to limitation. First of all, the heterogeneity in time until the taste test was carried out in the course of disease must be mentioned. A uniform timepoint of testing after onset of the GD and OD would be ideal but is difficult to be implemented into the daily hospital routine. After performing the questionnaire by telephone, the tests were sent by mail, which took at least two days. This method was chosen since it ensured a hygienic testing in home quarantine. To minimize the error, a telephone appointment was arranged with the patient and subjective findings on that day of testing again reviewed. While the number of participants in this cohort helps us to get first insights into the association of subjective and objective OD and GD in COVID-19 patients, further studies are needed in order to confirm our findings within a larger study population.

Acknowledgements The authors acknowledge Sofia Burgener and Vanessa Kley for their help with data collection.

Authors' contributions TSC conceived, designed and performed the study, performed statistical analysis, wrote and revised the manuscript and approved the final manuscript. JC performed statistical analysis, revised and approved the final manuscript. MO performed the study and approved the final manuscript. FUM performed the study, revised the manuscript and approved the final manuscript. SJB designed and performed the study, revised the manuscript and approved the final manuscript.

Funding Thirza Singer-Cornelius received funding from Kantonsspital Aarau, Department of Otolaryngology, funded by Research Council KSA 1410.000.128.

Data availability The data that support the findings of this study are available from the corresponding author, Thirza Singer-Cornelius, upon reasonable request.

Code availability Not applicable.

\section{Compliance with ethical standards}

Conflict of interest For all authors, there is no conflict of interest. The authors have no relevant financial or nonfinancial interests to disclose.

Human and animals rights The research involved human participants, no animals.

Informed consent Informed consent was obtained from all individual participants included in the study.

Consent to publish Patients signed informed consent regarding publishing their data. 


\section{References}

1. Lechien JR et al (2020) Olfactory and gustatory dysfunctions as a clinical presentation of mild-to-moderate forms of the coronavirus disease (COVID-19): a multicenter European study. Eur Arch Oto-Rhino-Laryngol 277:2251-2261

2. Speth MM et al (2020) Olfactory dysfunction and sinonasal symptomatology in COVID-19: prevalence, severity, timing, and associated characteristics. Otolaryngol Head Neck Surg 163:114-120

3. Han Q, Peng J, Xu H, Chen Q (2020) Taste cell is abundant in the expression of ACE2 receptor of 2019-nCoV. 2020040424. https ://doi.org/10.20944/preprints202004.0424.v1

4. Sungnak W et al (2020) SARS-CoV-2 entry factors are highly expressed in nasal epithelial cells together with innate immune genes. Nat Med. https://doi.org/10.1038/s41591-020-0868-6

5. Netland J et al (2008) Severe acute respiratory syndrome coronavirus infection causes neuronal death in the absence of encephalitis in mice transgenic for human ACE2. J Virol 82(15):7264-7275

6. Dube $\mathrm{M}$ et al (2018) Axonal transport enables neuron-to-neuron propagation of human coronavirus OC43. J Virol. https://doi. org/10.1128/JVI.00404-18

7. Giacomelli A et al (2020) Self-reported olfactory and taste disorders in patients with severe acute respiratory coronavirus 2 infection: a cross-sectional study. Clin Infect Dis. https://doi. org/10.1093/cid/ciaa330

8. Speth MM et al (2020) Time scale for resolution of olfactory dysfunction in COVID-19. Rhinology. https://doi.org/10.4193/Rhin2 0.227

9. Speth MM et al (2020) Mood, anxiety and olfactory dysfunction in COVID-19: evidence of central nervous system involvement? Laryngoscope. https://doi.org/10.1002/lary.28964

10. Moein ST et al (2020) Smell dysfunction: a biomarker for COVID19. Int Forum Allergy Rhinol 10:944-950

11 Lechien JR et al (2020) Objective olfactory evaluation of selfreported loss of smell in a case series of 86 COVID-19 patients. Head Neck. https://doi.org/10.1002/hed.26279

12. Doty RL, Frye RE, Agrawal U (1989) Internal consistency reliability of the fractionated and whole University of Pennsylvania smell identification test. Percept Psychophys 45(5):381-384
13. Landis BN et al (2009) "Taste Strips" - a rapid, lateralized, gustatory bedside identification test based on impregnated filter papers. J Neurol 256(2):242-248

14. Mueller C et al (2003) Quantitative assessment of gustatory function in a clinical context using impregnated "taste strips." Rhinology 41(1):2-6

15. Linder A (1988) Symptom scores as measures of the severity of rhinitis. Clin Allergy 18(1):29-37

16. Doty RL et al (1995) A study of the test-retest reliability of ten olfactory tests. Chem Senses 20(6):645-656

17. Wolf A et al (2016) A new extension to the Taste Strips test. Rhinology 54(1):45-50

18. Bachmanov AA et al (2014) Genetics of taste receptors. Curr Pharm Des 20(16):2669-2683

19. Shigemura N et al (2013) Angiotensin II modulates salty and sweet taste sensitivities. J Neurosci 33(15):6267-6277

20 Kai H, Kai M (2020) Interactions of coronaviruses with ACE2, angiotensin II, and RAS inhibitors-lessons from available evidence and insights into COVID-19. Hypertens Res 43:648-654

21. Challis RC, Ma M (2016) Sour taste finds closure in a potassium channel. Proc Natl Acad Sci 113(2):246

22 Jugdutt BI (2015) Suppression of ventricular arrhythmias after myocardial infarction by AT1 receptor blockade: role of the AT2 receptor and casein kinase 2/Kir2.1 pathway. Cardiovasc Drugs Therapy 29(3):201-206

23 Wu C et al (2020) Single-cell RNA expression profiling of ACE2, the putative receptor of Wuhan 2019-nCoV, in the nasal tissue. medRxiv 560:649

24. Zhou P et al (2020) A pneumonia outbreak associated with a new coronavirus of probable bat origin. Nature 579(7798):270-273

25. Li Y-C, Bai W-Z, Hashikawa T (2020) The neuroinvasive potential of SARS-CoV2 may play a role in the respiratory failure of COVID-19 patients. J Med Virol 92(6):552-555

Publisher's Note Springer Nature remains neutral with regard to jurisdictional claims in published maps and institutional affiliations. 